\title{
Boundary Eigenvalue Problem for Maxwell Equations in a Nonlinear Dielectric Layer
}

\author{
Yury G. Smirnov, Dmitry V. Valovik \\ Department Mathematics and Supercomputer Modeling, Penza State University, Penza, Russia \\ E-mail:smyrnovyug@mail.ru,dvalovik@mail.ru \\ Received March 4, 2010; revised April 16, 2010; accepted April 30, 2010
}

\begin{abstract}
The propagation of TM-polarized electromagnetic waves in a dielectric layer filled with lossless, nonmagnetic, and isotropic medium is considered. The permittivity in the layer defines by Kerr law. We look for eigenvalues of the problem and reduce the issue to the analysis of the corresponding dispersion equation. The equivalence of the boundary eigenvalue problem and the dispersion equation is proved. We show that the solution of the problem exists and from dispersion equation it can be numerically obtained. Using this solution the components of electromagnetic field in the layer can be numerically obtained as well. Transition to the limit in the case of a linear medium in the layer is proved. Some numerical results are presented also.
\end{abstract}

Keywords: Maxwell Equations, Nonlinear Boundary Eigenvalue Problem, Dispersion Equation, Nonlinear Slab (Film)

\section{Introduction}

Problems of electromagnetic waves propagation in a nonlinear media are actually important so as these phenomena are widely used in plasma physics, in modern microelectronics, in optics, in laser technology. Mathematical models for some of these problems and certain results are presented in [1-4]. These models yield boundary eigenvalue problems for systems of differential equations. These boundary eigenvalue problems depend on a spectral parameter nonlinearly. Analysis of these problems is very difficult for the reason that it is not possible to apply well-known methods of investigation of spectral problems.

It is necessary to note that such problems are exactly boundary eigenvalue problems. This is due to the fact that the main interest in the problems is finding that values of spectral parameter (eigenvalues) when the wave is propagating in the waveguide. Thus, in such problems it is necessary to pay attention on finding dispersion equations. When we have eigenvalues, the solutions of differential equations can be numerically obtained. With a mathematical view, a dispersion equation is an equation with respect to a spectral parameter, analyzing the equation we can deduce conclusion about existence of solutions of these boundary eigenvalue problems.

The phenomena of propagation of TE-waves were studied rather completely. Results of propagation TE waves in a nonlinear dielectric layer were presented in articles of H.-W. Shurmann, V. S. Serov and Yu. V.
Shestopalov [5,6]. Propagation of TE-waves in a nonlinear dielectric waveguide was considered in $[7,8]$ by $\mathrm{Yu}$. G. Smirnov, H.-W. Shurmann and Yu. V. Shestopalov. In both latter articles the problem is considered exactly like boundary eigenvalue problem (nonlinear, of course). Some numerical results are shown in [7]. The article [8] is devoted to strict mathematical results about the problem (solvability, existence of the solution, etc.).

Results about propagation of TM waves in a nonlinear dielectric semi-infinite layer were published in $[2,3]$. The first integral of the problem under consideration in the section 2 has been found in [4]. This integral represents the conservation law. However, the complete solution of the problem has not been found. The dispersion equation for the problem has not been obtained.

The equations of the TM waves propagation problem in nonlinear layer with Kerr nonlinearity were published for the first time in 1971-1972 in articles of P. N. Eleonskii and V. P. Silin (see, for example, [1]).

\section{Statement of the Problem}

Given a Cartesian system $O x y z$, consider electromagnetic waves propagating through a homogeneous isotropic nonmagnetic dielectric layer with Kerr nonlinearity located between two semi-infinite half-spaces $x<0$ and $x>h$. The half spaces are filled with isotropic nonmagnetic media without sources that have constant permittivities $\varepsilon_{1} \geq \varepsilon_{0}, \varepsilon_{3} \geq \varepsilon_{0}$, respectively, where 
$\varepsilon_{0}>0$ is the permittivity of vacuum. We suppose that in the whole space $\mu=\mu_{0}$; where $\mu_{0}$ is the permeability of vacuum.

The electric and magnetic fields are harmonic functions of time $t$ :

$$
\begin{aligned}
& \hat{\mathbf{E}}(x, y, z, t)=\mathbf{E}_{+}(x, y, z) \cos \omega t+\mathbf{E}_{-}(x, y, z) \sin \omega t \\
& \hat{\mathbf{H}}(x, y, z, t)=\mathbf{H}_{+}(x, y, z) \cos \omega t+\mathbf{H}_{-}(x, y, z) \sin \omega t
\end{aligned}
$$

and satisfy Maxwell equations

$$
\operatorname{rot} \mathbf{H}=-i \omega \varepsilon \mathbf{E}, \quad \operatorname{rot} \mathbf{E}=i \omega \mu \mathbf{H},
$$

where

$$
\begin{aligned}
\mathbf{E}(x, y, z) & =\mathbf{E}_{+}(x, y, z)+i \mathbf{E}_{-}(x, y, z) \\
\mathbf{H}(x, y, z) & =\mathbf{H}_{+}(x, y, z)+i \mathbf{H}_{-}(x, y, z)
\end{aligned}
$$

are complex amplitudes.

The permittivity inside the layer is described by the Kerr law $\varepsilon=\varepsilon_{2}+a|\mathbf{E}|^{2}$, where $\varepsilon_{2}>\max \left(\varepsilon_{1}, \varepsilon_{3}\right)$ and $a$ are positive constants. Here, $\mathbf{E}=\left(E_{x}, E_{y}, E_{z}\right)^{T}$, $|\mathbf{E}|^{2}=\left|E_{x}\right|^{2}+\left|E_{y}\right|^{2}+\left|E_{z}\right|^{2}$ and $|\cdot|$ is modulus of complex function. A solution to the Maxwell equations is sought in the entire space. Hereafter, the time multiplier is omitted.

The electromagnetic fields $\mathbf{E}$ and $\mathbf{H}$ satisfy Maxwell Equation (1), the condition that their tangential components at $x=0$ and $x=h$ are continuous, and the radiation condition at infinity; i.e., the electromagnetic field decays exponentially as $|x| \rightarrow \infty$ in the regions $x<0$ and $x>h$.

Consider the TM waves $\mathbf{E}=\left(E_{x}, 0, E_{z}\right)^{T}$ and $\mathbf{H}=\left(0, H_{y}, 0\right)^{T}$. As a result, Equation (1) become

$$
\left\{\begin{array}{l}
\frac{\partial E_{z}}{\partial y}=0 ; \quad \frac{\partial E_{x}}{\partial z}-\frac{\partial E_{z}}{\partial x}=i \omega \mu H_{y} ; \quad \frac{\partial E_{x}}{\partial y}=0 \\
\frac{\partial H_{y}}{\partial z}=i \omega \varepsilon E_{x} ; \quad \frac{\partial H_{y}}{\partial x}=-i \omega \varepsilon E_{z} .
\end{array}\right.
$$

It follows from (2) that $E_{z}=E_{z}(x, z)$ and $E_{x}=E_{x}(x, z)$ are independent of $y$. Since $H_{y}$ is expressed in terms of $E_{x}$ and $E_{z}$, we conclude that $H_{y}$ is also independent of $y$.

Let $\frac{\partial}{\partial x} \equiv(\ldots)^{\prime}$. Assuming that the field components depend harmonically on $z$, i.e., $H_{y}=H_{y}(x, z)=$ $H_{y}(x) e^{i \gamma z}, \quad E_{x}=E_{x}(x, z)=E_{x}(x) e^{i \gamma z}, \quad$ and $E_{z}=$ $E_{z}(x, z)=E_{z}(x) e^{i \gamma z}$, we obtain the system of equations

$$
\left\{\begin{array}{l}
i \gamma E_{x}(x)-E_{z}^{\prime}(x)=i \omega \mu H_{y}(x) \\
H_{y}^{\prime}(x)=-i \omega \varepsilon E_{z}(x) \\
i \gamma H_{y}(x)=i \omega \varepsilon E_{x}(x)
\end{array}\right.
$$

which implies that

$$
H_{y}(x)=\frac{1}{i \omega \mu}\left(i \gamma E_{x}(x)-E_{z}^{\prime}(x)\right) .
$$

Here $\gamma$ is unknown spectral parameter, i.e., the propagation constant.

Differentiating (4) and using (3) yields

$$
\left\{\begin{array}{l}
\gamma\left(i E_{x}(x)\right)^{\prime}-E_{z}^{\prime \prime}(x)=\omega^{2} \varepsilon \mu E_{z}(x) ; \\
\gamma^{2}\left(i E_{x}(x)\right)-\gamma E_{z}^{\prime}(x)=\omega^{2} \varepsilon \mu\left(i E_{x}(x)\right) .
\end{array}\right.
$$

Introducing $k^{2}=\omega^{2} \mu \varepsilon_{0}$ with $\mu=\mu_{0}$ and normalizing the equations according to the formulas $\tilde{x}=k x$, $\frac{d}{d x}=k \frac{d}{d \tilde{x}}, \quad \tilde{\gamma}=\frac{\gamma}{k}, \quad \tilde{\varepsilon}_{j}=\frac{\varepsilon_{j}}{\varepsilon_{0}} \quad(j=1,2,3), \quad \tilde{a}=\frac{a}{\varepsilon_{0}}$.

We introduce the new notation $E_{z} \equiv Z(\tilde{x})$ and $i E_{x} \equiv X(\tilde{x})$.

By omitting the tilde, (5) is written in the normalized form as

$$
\left\{\begin{array}{l}
-Z^{\prime \prime}+\gamma X^{\prime}=\varepsilon Z \\
-\gamma Z^{\prime}+\gamma^{2} X=\varepsilon X
\end{array}\right.
$$

The real solution $X(x)$ and $Z(x)$ of (6) are sought assuming that $\gamma$ is real (so that $|\mathbf{E}|^{2}$ is independent of $z)$, where

$$
\varepsilon=\left\{\begin{array}{l}
\varepsilon_{1}, x<0 \\
\varepsilon_{2}+a\left(X^{2}+Z^{2}\right), 0<x<h \\
\varepsilon_{3}, x>h
\end{array}\right.
$$

It is also assumed that $X(x)$ and $Z(x)$ are differentiable in the layer:

$$
\begin{aligned}
& X(x) \in C(-\infty ; 0] \cap C[0 ; h] \cap[h ;+\infty) \cap \\
& \cap C^{1}(-\infty ; 0) \cap C^{1}(0 ; h) \cap C^{1}(h ;+\infty) ; \\
& Z(x) \in C(-\infty ;+\infty) \cap \\
& \cap C^{1}(-\infty ; 0] \cap C^{1}[0 ; h] \cap C^{1}[h ;+\infty) \cap \\
& \cap C^{2}(-\infty ; 0) \cap C^{2}(0 ; h) \cap C^{2}(h ;+\infty) .
\end{aligned}
$$

These smoothness conditions follow from continuous conditions of tangential components at $x=0$ and $x=h$. We search for $\gamma$ such that $\max \left(\varepsilon_{1}, \varepsilon_{3}\right)<\gamma^{2}<\varepsilon_{2}$.

The statement of the problem is shown in the Figure 1. 


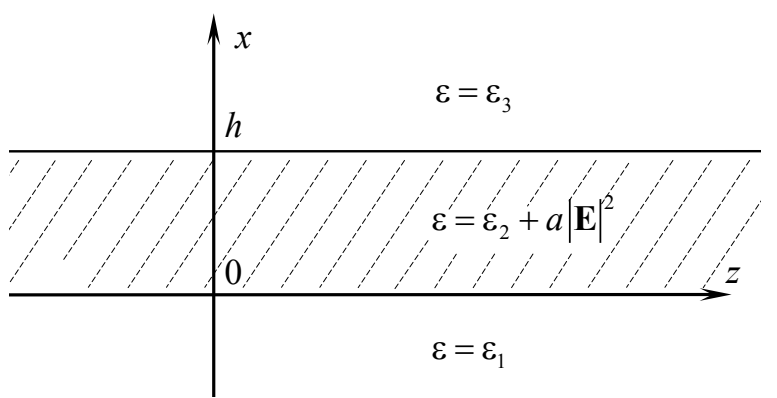

Figure 1. The geometry of the problem.

\section{Solution to the System of Differential Equations}

For $\varepsilon=\varepsilon_{1}$ in the half-space $x<0$, the general solution of (6) is

$$
\left\{\begin{array}{l}
X(x)=A \exp \left(x \sqrt{\gamma^{2}-\varepsilon_{1}}\right), \\
Z(x)=\frac{\sqrt{\gamma^{2}-\varepsilon_{1}}}{\gamma \sqrt{ }} A \exp \left(x \sqrt{\gamma^{2}-\varepsilon_{1}}\right),
\end{array}\right.
$$

where we took into account the condition at infinity.

For $\varepsilon=\varepsilon_{3}$ in the half-space $x>h$, we have

$$
\left\{\begin{array}{l}
X(x)=B \exp \left(-(x-h) \sqrt{\gamma^{2}-\varepsilon_{3}}\right), \\
Z(x)=-\frac{\sqrt{\gamma^{2}-\varepsilon_{3}}}{\gamma} B \exp \left(-(x-h) \sqrt{\gamma^{2}-\varepsilon_{3}}\right),
\end{array}\right.
$$

according to the condition at infinity. The constants $A$ and $B$ in (8) and (9) are determined by the boundary conditions.

Inside the layer $0<x<h$, (6) becomes

$$
\left\{\begin{array}{l}
-\frac{d^{2} Z}{d x^{2}}+\gamma \frac{d X}{d x}=\left(\varepsilon_{2}+a\left(X^{2}+Z^{2}\right)\right) Z ; \\
-\frac{d Z}{d x}+\gamma X=\frac{1}{\gamma}\left(\varepsilon_{2}+a\left(X^{2}+Z^{2}\right)\right) X .
\end{array}\right.
$$

We can reduce (10) to the form:

$$
\left\{\begin{array}{l}
\frac{d X}{d x}=\frac{2 a}{\gamma} \frac{\varepsilon_{2}-\gamma^{2}+a\left(X^{2}+Z^{2}\right)}{\varepsilon_{2}+3 a X^{2}+a Z^{2}} X^{2} Z+ \\
+\gamma \frac{\varepsilon_{2}+a\left(X^{2}+Z^{2}\right)}{\varepsilon_{2}+3 a X^{2}+a Z^{2}} Z ; \\
\frac{d Z}{d x}=-\frac{1}{\gamma}\left(\varepsilon_{2}-\gamma^{2}+a\left(X^{2}+Z^{2}\right)\right) X .
\end{array}\right.
$$

From (11) we have an ordinary differential equation:

$$
-\left(\varepsilon_{2}+3 a X^{2}+a Z^{2}\right) \frac{d X}{d Z}=
$$

$$
=2 a X Z+\gamma^{2} \frac{\varepsilon_{2}+a\left(X^{2}+Z^{2}\right)}{\varepsilon_{2}-\gamma^{2}+a\left(X^{2}+Z^{2}\right)} \frac{Z}{X}
$$

Multiplying (12) by $\left(\varepsilon_{2}-\gamma^{2}+a\left(X^{2}+Z^{2}\right)\right) X$ we obtain a total ordinary differential equation. Its solution is easy to write in the form:

$$
\begin{gathered}
\frac{\gamma^{6} C_{1}+3 \gamma^{2}\left(\varepsilon_{2}+a\left(X^{2}+Z^{2}\right)\right)^{2}-2\left(\varepsilon_{2}+a\left(X^{2}+Z^{2}\right)\right)^{3}}{2\left(\varepsilon_{2}+a\left(X^{2}+Z^{2}\right)\right)\left(2 \gamma^{2}-\left(\varepsilon_{2}+a\left(X^{2}+Z^{2}\right)\right)\right)}= \\
=a Z^{2}+\varepsilon_{2}
\end{gathered}
$$

from item 2 it is known that $\gamma^{2}>\varepsilon_{0}$ and $a>0$. Equation (13) is true under these conditions.

Define the new variables

$\tau(x)=\frac{\varepsilon_{2}+a\left(X^{2}(x)+Z^{2}(x)\right)}{\gamma^{2}}, \eta(x)=\gamma \frac{X(x)}{Z(x)} \tau(x)$.

Let $\tau_{0}=\frac{\varepsilon_{2}}{\gamma^{2}}$, then $X^{2}=\frac{\gamma^{2}}{a} \frac{\eta^{2}\left(\tau-\tau_{0}\right)}{\eta^{2}+\gamma^{2} \tau^{2}}$,

$Z^{2}=\frac{\gamma^{4}}{a} \frac{\tau^{2}\left(\tau-\tau_{0}\right)}{\eta^{2}+\gamma^{2} \tau^{2}}$. In these variables, (11) and (13) become

$$
\left\{\begin{aligned}
\frac{d \tau}{d x} & =2 \gamma^{2} \frac{\tau^{2} \eta\left(\tau-\tau_{0}\right)(2-\tau)}{\tau\left(\eta^{2}+\gamma^{2} \tau^{2}\right)+2 \eta^{2}\left(\tau-\tau_{0}\right)}, \\
\frac{d \eta}{d x} & =\frac{\gamma^{2} \tau^{2}+\eta^{2}(\tau-1)}{\tau} ; \\
\eta^{2} & =\frac{\gamma^{2} \tau^{2}\left(\tau^{2}-C_{1}\right)}{C_{1}+3 \tau^{2}-2 \tau^{3}-2 \tau(2-\tau) \tau_{0}} .
\end{aligned}\right.
$$

Here, (16) is a fourth-degree algebraic equation in $\tau$. Its solution $\tau=\tau(\eta)$ can be explicitly written using the Cardano-Ferrari formulas (see [9]).

\section{Boundary Conditions and Dispersion Equation}

To derive dispersion equations for the propagation constants, we have to find $\eta(0)$ and $\eta(h)$.

Since the tangential components of $\mathbf{E}$ and $\mathbf{H}$ are continuous, we obtain

$$
\begin{gathered}
Z(h)=E_{z}(h+0)=E_{z}^{(h)}, Z(0)=E_{z}(0-0)=E_{z}^{(0)} \\
\gamma X(h)-Z^{\prime}(h)=i \omega \mu H_{y}(h+0)=H_{y}^{(h)},
\end{gathered}
$$


$\gamma X(0)-Z^{\prime}(0)=i \omega \mu H_{y}(0)=H_{y}^{(0)}$, where $E_{z}^{(h)}$ is a known constant. Then

$$
H_{y}^{(h)}=-E_{z}^{(h)} \frac{\varepsilon_{3}}{\sqrt{\gamma^{2}-\varepsilon_{3}}}, \quad H_{y}^{(0)}=E_{z}^{(0)} \frac{\varepsilon_{1}}{\sqrt{\gamma^{2}-\varepsilon_{1}}} .
$$

According to (7) in the layer, we have

$-Z^{\prime}(x)+\gamma X(x)=\frac{1}{\gamma}\left(\varepsilon_{2}+a\left(X^{2}(x)+Z^{2}(x)\right)\right) X(x)$.

Combining (14), (16), (17), and (19) yields

$$
\begin{gathered}
\frac{X^{2}(h)}{\left(E_{z}^{(h)}\right)^{2}}=\frac{\tau^{2}(h)-C_{1}}{C_{1}+3 \tau^{2}(h)-2 \tau^{3}(h)-2 \tau(h)(2-\tau(h)) \tau_{0}} \\
\frac{1}{\gamma}\left(\varepsilon_{2}+a\left(X^{2}(h)+\left(E_{z}^{(h)}\right)^{2}\right)\right) X(h)=H_{y}^{(h)}
\end{gathered}
$$

where

$$
X(h)=\frac{H_{y}^{(h)}}{\gamma \tau(h)} .
$$

Solving (21) for $X(h)$, we obtain

$$
X^{3}(h)+\frac{\varepsilon_{2}+a\left(E_{z}^{(h)}\right)^{2}}{a} X(h)-\frac{\gamma H_{y}^{(h)}}{a}=0 .
$$

Since the value of $\left(\varepsilon_{2}+a\left(E_{z}^{(h)}\right)^{2}\right) / a$ is nonnegative, (23) has at least one real root (which we consider).

Thus, $\tau(h)=H_{y}^{(h)} /(\gamma X(h))$. Using (18) and (22), we find

$$
X(h)=-\frac{E_{z}^{(h)}}{\gamma \tau(h)} \frac{\varepsilon_{3}}{\sqrt{\gamma^{2}-\varepsilon_{3}}} .
$$

Combining (20) with (24) gives

$$
C_{1}=\tau^{2}(h)-\frac{2 \varepsilon_{3}^{2} \tau(h)(2-\tau(h))\left(\tau(h)-\tau_{0}\right)}{\varepsilon_{3}^{2}+\gamma^{2}\left(\gamma^{2}-\varepsilon_{3}\right) \tau^{2}(h)} .
$$

If $C_{1}>0$, then (16) which is regarded as an equation in $\tau(h)$ has a positive root. It is easy to show that $C_{1}$ is strictly positive. Indeed, (25) implies that $C_{1}>0$ for $\tau(h)>2$, since $\tau(h) \geq \tau_{0}>1$ and $\left(\gamma^{2}-\varepsilon_{3}\right)>0$. Consider the case of $\tau(h) \in\left[\tau_{0}, 2\right)$. Converting the terms in (25) to a common denominator and, if necessary, making the substitution $\tau(h)=\tau_{0}+\alpha \quad(0<\alpha<1)$, we obtain

$$
C_{1}=\tau(h) \frac{\gamma^{2}\left(\gamma^{2}-\varepsilon_{3}\right) \tau^{3}(h)+\varepsilon_{3}^{2}\left(2 \alpha(\tau(h)-1)+\tau_{0}-\alpha\right)}{\varepsilon_{3}^{2}+\gamma^{2}\left(\gamma^{2}-\varepsilon_{3}\right) \tau^{2}(h)}
$$

with a positive right-hand side.
It is well known that the field components $\varepsilon X(x)$ and $Z(x)$ are continuous at the interface of the media. Then, the function $\gamma \frac{\tau(x) X(x)}{Z(x)}$ is also continuous at the interface of the media at points $x$ such that $Z(x) \neq 0$. Since $\eta(x)=\gamma \frac{\tau(x) X(x)}{Z(x)}$, we use (8) and (9) to obtain

$$
\eta(0)=\frac{\varepsilon_{1}}{\sqrt{\gamma^{2}-\varepsilon_{1}}}>0 ; \eta(h)=-\frac{\varepsilon_{3}}{\sqrt{\gamma^{2}-\varepsilon_{3}}}<0 .
$$

Since the right-hand side of the second equation in (15) is positive, $\eta(x)$ is an increasing function on the interval $(0 ; h)$. Taking into account the signs in $(26)$, we conclude that $\eta(x)$ is not differentiable on the entire interval $(0 ; h)$ but has a point of discontinuity. Assume that this point is $x^{*} \in(0 ; h)$. It follows from (16) that $x^{*}$ is such that $\tau^{*}=\tau\left(x^{*}\right)$ is a root of the equation $C_{1}+3\left(\tau^{*}\right)^{2}-2\left(\tau^{*}\right)^{3}-2 \tau^{*}\left(2-\tau^{*}\right) \tau_{0}=0 . \quad$ Moreover, $\eta\left(x^{*}-0\right) \rightarrow+\infty$ and $\eta\left(x^{*}+0\right) \rightarrow-\infty$. Let

$$
f \equiv f(\eta)=\frac{\tau}{\gamma^{2} \tau^{2}+\eta^{2}(\tau-1)},
$$

where $\tau=\tau(\eta)$ is expressed from (16). In the general case, there are several points $x_{0}, x_{1}, \ldots, x_{N}$ on the interval $[0, h]$ at which $\eta(x)$ becomes infinite, so that

$$
\begin{aligned}
& \eta\left(x_{0}-0\right)=\eta\left(x_{1}-0\right)=\ldots=\eta\left(x_{N}-0\right)=+\infty, \\
& \eta\left(x_{0}+0\right)=\eta\left(x_{1}+0\right)=\ldots=\eta\left(x_{N}+0\right)=-\infty .
\end{aligned}
$$

Below, it will be proved that the number of such points is finite for any $h$.

Solutions are sought on each of the intervals $\left[0, x_{0}\right]$, $\left[x_{0}, x_{1}\right], \ldots,\left[x_{N}, h\right]$ :

$$
\begin{gathered}
-\int_{\eta(x)}^{\eta\left(x_{0}\right)} f d \eta=x+c_{0}, \quad 0 \leq x \leq x_{0} ; \\
\int_{\eta\left(x_{i}\right)}^{\eta(x)} f d \eta=x+c_{i+1}, \quad x_{i} \leq x \leq x_{i+1}, \quad i=\overline{0, N-1} ; \\
\int_{\eta\left(x_{N}\right)}^{\eta(x)} f d \eta=x+c_{N+1}, \quad x_{N} \leq x \leq h .
\end{gathered}
$$

Taking into account (27) and substituting $x=0$, $x=x_{i+1}, x=x_{N}$ into the first, second, and third equations in (28), respectively, we find the required constants $c_{1}, c_{2}, \ldots, c_{N+1}$ : 


$$
\begin{gathered}
c_{0}=-\int_{\eta(0)}^{+\infty} f d \eta ; \\
c_{i+1}=\int_{-\infty}^{+\infty} f d \eta-x_{i+1}, \quad i=\overline{0, N-1} ; \\
c_{N+1}=\int_{-\infty}^{\eta(h)} f d \eta-h .
\end{gathered}
$$

In view of (29) Equation (28) become

$$
\begin{gathered}
\int_{\eta(x)}^{\eta\left(x_{0}\right)} f d \eta=-x+\int_{\eta(0)}^{+\infty} f d \eta, \quad 0 \leq x \leq x_{0} \\
\int_{\eta\left(x_{i}\right)}^{\eta(x)} f d \eta=x+\int_{-\infty}^{+\infty} f d \eta-x_{i+1}, \quad x_{i} \leq x \leq x_{i+1}, \quad i=\overline{0, N-1} \\
\int_{\eta\left(x_{N}\right)}^{\eta(x)} f d \eta=x+\int_{-\infty}^{\eta(h)} f d \eta-h, \quad x_{N} \leq x \leq h
\end{gathered}
$$

Let $\int_{-\infty}^{+\infty} f d \eta=T$. It follows from (30) that $x_{i+1}-x_{i}=$ $T>0$, where $i=\overline{0, N-1}$. Therefore, the number of points at which $\eta(x)$ becomes infinite on the interval $(0 ; h)$ is finite. Now, setting $x=x_{0}, x=x_{i}, x=x_{N}$ in the first, second, and third equations in (30), respectively, so that all the integrals on the left-hand sides vanish, we add all the equations in (30) to obtain

$$
\begin{aligned}
0 & =-x_{0}+\int_{\eta(0)}^{+\infty} f d \eta+x_{0}+T-x_{1}+\ldots+x_{N-1}+ \\
& +T-x_{N}+x_{N}+\int_{-\infty}^{\eta(h)} f d \eta-h
\end{aligned}
$$

Finally, (31) yields

$$
-\int_{-\frac{\varepsilon_{3}}{\sqrt{\gamma^{2}-\varepsilon_{3}}}}^{\frac{\varepsilon_{1}}{\sqrt{\gamma^{2}-\varepsilon_{1}}}} f d \eta+(N+1) T=h,
$$

where $N \geq 0$ is an integer.

Formula (32) is a dispersion equation that holds for any $h$. It should be noted that, if $N \neq 0$, we have several equations for various values of $N$. Each of these equations must be solved for $\gamma$. All the resulting $\gamma$ form a set of the propagation constants for which and only for which waves in the layer propagate for given $h$.

It should be also noted that $\int_{-\infty}^{+\infty} f d \eta$ converges since $f$ can be majorized by the function $\frac{M}{(m-1) \eta^{2}}$, where $M=\max _{x \in[0, h]} \tau(x)$, and $m=\min _{x \in[0, h]} \tau(x)>1$. Since $X(x)$ and $Z(x)$ are bounded, $\tau(x)$ has a finite minimum and maximum.

If we consider first equation of (15) combined with first integral, then the equation can be integrated. The obtained integral is so called hyperelliptic integral (it is one of the simplest type of Abelian integrals). If we extend definitional domain of independent variable $x$ on the whole complex plane, then we can consider the inverse function for these integrals. These functions will be solutions of the system (15). These functions are hyperelliptic functions which belong to set Abelian functions. Abelian functions are meromorphic periodical functions. So as function $\eta$ algebraically depends on $\tau$ therefore $\eta$ is a meromorphic periodical function. Thus, the break point $x^{*}$ is a one of the poles of function $\eta$. The integral in (32) is a more general Abelian integral [10, $11]$.

\section{Boundary Value Problem and Existence Theorems}

The continuity conditions for components of field $\mathbf{E}$ imply

$$
[\varepsilon X]_{x=0}=0,[\varepsilon X]_{x=h}=0,[Z]_{x=0}=0,[Z]_{x=h}=0 \text {. }
$$

We suppose that the functions $X(x)$ and $Z(x)$ also satisfy the conditions

$$
X(x)=O\left(\frac{1}{|x|}\right) \text { and } Z(x)=O\left(\frac{1}{|x|}\right) \text { as }|x| \rightarrow \infty .
$$

Introduce the notation

$$
\begin{aligned}
& \mathbf{D}=\left(\begin{array}{cc}
d / d x & 0 \\
0 & d / d x
\end{array}\right), \\
& \mathbf{F}(X(x), Z(x))=\left(\begin{array}{l}
X(x) \\
Z(x)
\end{array}\right), \quad \mathbf{G}(F, \gamma)=\left(\begin{array}{l}
G_{1}(F, \gamma) \\
G_{2}(F, \gamma)
\end{array}\right),
\end{aligned}
$$

where $X(x)$ and $Z(x)$ are unknown functions, $G_{1}$ and $G_{2}$ are left-hand sides of system (11). The $\gamma$ is a spectral parameter. Also we will be considered column-vector $\mathbf{N}(x)=\left(\begin{array}{c}\varepsilon X(x) \\ Z(x)\end{array}\right)$. Rewrite the problem using introduced notation.

In the half-space $x<0$ and $\varepsilon=\varepsilon_{1}$, we have $\mathbf{N}=\left(\begin{array}{c}\varepsilon_{1} X \\ Z\end{array}\right)$ and

$$
\gamma \mathbf{D F}-\left(\begin{array}{cc}
0 & \gamma^{2} \\
\gamma^{2}-\varepsilon_{1} & 0
\end{array}\right) \mathbf{F}=0 \text {. }
$$

Inside the layer $0<x<h$ and $\varepsilon=\varepsilon_{2}+a|\mathbf{E}|^{2}$, we 
have $\mathbf{N}=\left(\begin{array}{c}\left(\varepsilon_{2}+a\left(X^{2}+Z^{2}\right)\right) X \\ Z\end{array}\right)$, and the system has the form

$$
\mathbf{L}(F, \gamma) \equiv \mathbf{D F}-\mathbf{G}(F, \gamma)=0 .
$$

In the half-space $x>h$ and $\varepsilon=\varepsilon_{3}$, we have $\mathbf{N}=\left(\begin{array}{c}\varepsilon_{3} X \\ Z\end{array}\right)$ and

$$
\gamma \mathbf{D F}-\left(\begin{array}{cc}
0 & \gamma^{2} \\
\gamma^{2}-\varepsilon_{3} & 0
\end{array}\right) \mathbf{F}=0 .
$$

The continuity conditions (33) imply the following conditions

$$
[\mathbf{N}(x)]_{x=0}=0,[\mathbf{N}(x)]_{x=h}=0,
$$

where $[f(x)]_{x=x_{0}}=\lim _{x \rightarrow x_{0}-0} f(x)-\lim _{x \rightarrow x_{0}+0} f(x)$, for vector it means transition to the limit for every components of the vector.

Let us formulate boundary problem (conjugation problem). We will search for non-vanishing vector $F$ and corresponding eigenvalues $\gamma$ so that $F$ satisfies (35)-(37) and conjugation conditions (38). In addition the components of the vector $F$ must obey the condition (34).

The function $\mathbf{L}(F, \gamma)$ from (36) is a nonlinear operator-function, which nonlinearly depends on the spectral parameter. Spectral theory of linear operator-functions, which nonlinearly depend on spectral parameter was built in [12]. As yet there is not a common spectral theory of nonlinear operator-functions which nonlinearly depend on spectral parameter. Therefore, commonly boundary value problems with these operator-functions can not be solved by known methods.

Definition 1. The value $\gamma=\gamma_{0}$ we call eigenvalue of the problem if the problem (35)-(37) with conditions (34) and (38) has unique nonzero solution $\mathbf{F}$. The solution $\mathbf{F}$, which corresponds to eigenvalues $\gamma_{0}$ we call eigenvector of the problem, and the components $X(x)$ and $Z(x)$ of the eigenvector $\mathbf{F}$ we call eigenfunctions.

There is a well-known definition of eigenvalue of linear operator-function which nonlinear depends from spectral parameter [12]. Definition 1 is a non-classical analog of that well-known definition. Definition under consideration, on the one hand, is a distribution of classical definition for a case of nonlinear operator-function nonlinearly depending on spectral parameter. On the other hand, definition 1 corresponds to physical statement of the problem.

Theorem 1. Boundary value problem (35)-(37) with conditions (34) and (38) has a solution (eigenvalue) then and only then, when the eigenvalue is a solution of dispersion Equation (32).

Proof. Sufficiency. It is obviously, that, if we have a solution $\gamma$ of dispersion Equation (32), we can find functions $\tau(x)$ and $\eta(x)$ from system (15) and first integral (16). Using Formula (14), we obtain

$$
\begin{aligned}
& X(x)= \pm \frac{\gamma \eta}{\sqrt{a}} \sqrt{\frac{\tau-\tau_{0}}{\eta^{2}+\gamma^{2} \tau^{2}}} \\
& Z(x)= \pm \frac{\gamma^{2} \tau}{\sqrt{a}} \sqrt{\frac{\sqrt{1-\tau_{0}}}{\eta^{2}}} \cdot
\end{aligned}
$$

The question of sign's chøosipg is very important. We know the behavior of function $\eta=\gamma \tau \frac{X}{Z}$ : the function $\eta$ is monotone increasing, if $x=x^{*}$ is such that $\eta\left(x^{*}\right)=0$, then $\eta\left(x^{*}-0\right)<0, \eta\left(x^{*}+0\right)>0$, and if $x=x^{* *}$ is such that $\eta\left(x^{* *}\right)= \pm \infty$, then $\eta\left(x^{* *}-0\right)>0$, $\eta\left(x^{* *}+0\right)<0$. Function $\eta$ has not other points of sign reversal. The boundary conditions result in $Z(h)=E_{z}^{(h)}(>0)$. We know, if $\eta>0$, then functions $X$ and $Z$ have the same signs, but if $\eta<0$, then $X$ and $Z$ have opposite signs, and keep in mind that $X$ and $Z$ are continuously differentiable functions (in corresponding domains), we chose suitable signs in expressions (39).

Necessity. The way of obtaining dispersion Equation (32) from (15) implies that the eigenvalue of the problem is a solution of dispersion equation.

It is necessary to note that the eigenfunctions correspond to eigenvalue $\gamma_{0}$ can easily find from (11) by using, for example, Runge-Kutta method.

Let us formulate existence and localization theorem is founded on the obtained results. Introduce the notation $J \equiv J(a, \gamma, N)$ which denotes the right-hand side of dispersion Equation (32). It is clear that for each integer nonnegative finite $N$

$$
\begin{gathered}
\inf _{\gamma^{2} \in\left(\max \left(\varepsilon_{1}, \varepsilon_{3}\right), \varepsilon_{2}\right)} J(a, \gamma, N)>0, \\
\sup _{\gamma^{2} \in\left(\max \left(\varepsilon_{1}, \varepsilon_{3}\right), \varepsilon_{2}\right)} J(a, \gamma, N)<\infty .
\end{gathered}
$$

Moreover, decreasing of $N$ implies decreasing of the infimum and supremum, and increasing of $N$ implies increasing of the infimum and supremum. It is obviously from the form of dispersion equation.

Theorem 2. Let

$$
h_{1}^{(k)}=\inf _{\gamma^{2} \in\left(\max \left(\varepsilon_{1}, \varepsilon_{3}\right), \varepsilon_{2}\right)} J(a, \gamma, k),
$$




$$
h_{2}^{(k)}=\sup _{\gamma^{2} \in\left(\max \left(\varepsilon_{1}, \varepsilon_{3}\right), \varepsilon_{2}\right)} J(a, \gamma, k)
$$

and $h$ is such that there are $i$ and $j$, that $h_{2}^{(i)}<h$ and $h_{2}^{(i+1)}>h ; h_{1}^{(j)}<h$ and $h_{1}^{(j+1)}>h$.

Then, there are at least $j-i$ eigenvalues of the problem (35)-(37) with conditions (34) and (38).

Theorem 2 requires some explanations. If for some $j$ infimum is greater than $h$ then for all greater numbers $j$ infimum is all the more greater than $h$ and dispersion equation has not solutions. Similarly, if for some $i$ supremum is smaller than $h$, then for all smaller numbers $i$ supremum is all the more smaller than $h$. In this case dispersion equation also has not solutions. These explanations follow from the fact that the infimum and supremum of left-hand of dispersion Equation (32) are finite.

\section{Transition to the Limit in the Case of a Linear Medium in the Layer}

Consider formal transition to the limit as $a \rightarrow 0$ in the case of a linear medium in the layer. The dispersion equation in the linear case is (see [13]):

$$
\operatorname{tg}\left(h \sqrt{\varepsilon_{2}-\gamma^{2}}\right)=\frac{\varepsilon_{2} \sqrt{\varepsilon_{2}-\gamma^{2}}\left(\varepsilon_{1} \sqrt{\gamma^{2}-\varepsilon_{3}}+\varepsilon_{3} \sqrt{\gamma^{2}-\varepsilon_{1}}\right)}{\varepsilon_{1} \varepsilon_{3}\left(\varepsilon_{2}-\gamma^{2}\right)-\varepsilon_{2}^{2} \sqrt{\gamma^{2}-\varepsilon_{3}} \sqrt{\gamma^{2}-\varepsilon_{1}}}
$$

Consider the functions

$$
f=\frac{\tau}{\gamma^{2} \tau^{2}+\eta^{2}(\tau-1)} \text { and } f_{1}=\frac{\varepsilon_{2}}{\varepsilon_{2}-\gamma^{2}} \frac{1}{\frac{\varepsilon_{2}^{2}}{\varepsilon_{2}-\gamma^{2}}+\eta^{2}} .
$$

The function $f_{1}$ is derived from $f$ by formal transition to the limit as $a \rightarrow 0$ with respect to $\tau$. Since we search for real solutions $X(x)$ and $Z(x)$, the denominator of $f_{1}$ cannot vanish. Moreover, as $a \rightarrow 0$, the function $f$ tends to $f_{1}$ uniformly with respect to $x \in[0, h]$. Under this condition, since $f$ is continuous, we can use classical calculus result to transit to the limit as $a \rightarrow 0$ under the integral sign in (32):

$$
\begin{gathered}
\left(-\int_{-\frac{\varepsilon_{3}}{\sqrt{\gamma^{2}-\varepsilon_{3}}}}^{\frac{\varepsilon_{1}}{\sqrt{\gamma^{2}-\varepsilon_{1}}}} \frac{1}{\frac{\varepsilon_{2}^{2}}{\varepsilon_{2}-\gamma^{2}}+\eta^{2}} d \eta+(N+1) \int_{-\infty}^{+\infty} \frac{1}{\frac{\varepsilon_{2}^{2}}{\varepsilon_{2}-\gamma^{2}}+\eta^{2}} d \eta\right)= \\
=\frac{\varepsilon_{2}-\gamma^{2}}{\varepsilon_{2}} h
\end{gathered}
$$

The integrals in (41) are analytically evaluated. Finally, (41) yields

$$
\begin{gathered}
h \sqrt{\varepsilon_{2}-\gamma^{2}}=\operatorname{arctg} \frac{\varepsilon_{2} \sqrt{\varepsilon_{2}-\gamma^{2}}\left(\varepsilon_{1} \sqrt{\gamma^{2}-\varepsilon_{3}}+\varepsilon_{3} \sqrt{\gamma^{2}-\varepsilon_{1}}\right)}{\varepsilon_{1} \varepsilon_{3}\left(\varepsilon_{2}-\gamma^{2}\right)-\varepsilon_{2}^{2} \sqrt{\gamma^{2}-\varepsilon_{3}} \sqrt{\gamma^{2}-\varepsilon_{1}}}+ \\
+(N+1) \pi
\end{gathered}
$$

Taking the tangent of (42) gives (40).

Results of this paragraph show us that we obtain regular case when we transit to the limit as $a \rightarrow 0$. The limit of dispersion Equation (32) for nonlinear medium leads to dispersion Equation (40) for linear medium. The dispersion Equation (40) is well-known classical result in electrodynamics.

Note that the method of finding dispersion equation considered in this section can be applied to more general problem. Namely, to the problem of propagation TM wave in an anisotropic nonlinear layer with Kerr nonlinearity (we studied a case of isotropic nonlinear layer). The statement of the problem differs only one detail. In a case of anisotropic nonlinear layer the permittivity is described by diagonal tensor $\widehat{\varepsilon}=\left(\begin{array}{ccc}\varepsilon_{x x} & 0 & 0 \\ 0 & \varepsilon_{y y} & 0 \\ 0 & 0 & \varepsilon_{z z}\end{array}\right)$, where $\varepsilon_{x x}=\varepsilon_{12}+b\left|E_{x}\right|^{2}+a\left|E_{z}\right|^{2}, \quad \varepsilon_{z z}=\varepsilon_{21}+a\left|E_{x}\right|^{2}+b\left|E_{z}\right|^{2}, a$, $b$ are nonlinearity coefficients and $\varepsilon_{12}>\max \left(\varepsilon_{1}, \varepsilon_{3}\right)$ and $\varepsilon_{21}>\max \left(\varepsilon_{1}, \varepsilon_{3}\right)$. Here, after writing the system of equation in terms of functions $X(x)$ and $Z(x)$ we have to chose new variables $\tau(x)$ and $\eta(x)$ in form $\tau(x)=\frac{\varepsilon_{12}+b X^{2}+a Z^{2}}{\gamma^{2}}$ and $\eta(x)=\gamma \tau \frac{X}{Z}$, where $X=X(x)$ and $Z=Z(x)$.

Anisotropic case with additional condition $\varepsilon_{12}=$ $\varepsilon_{21}=\varepsilon_{2}$ was completely investigated in [14].

The first approximation for the propagation constants was presented in [15].

\section{Numerical Results}

In this section some numerical results are presented. The calculations are illustrated by the plots.

In Figure 2, the solid lines show the solutions to the dispersion equation for the case of a linear medium in the layer, and the dashed lines correspond to the nonlinear dispersion equation.

Figure 2 illustrates the dependence of squared propagation constant $\gamma^{2}$ on layer's thickness $h$. The following parameters are used: $\varepsilon_{1}=\varepsilon_{3}=1, \varepsilon_{2}=9$, 


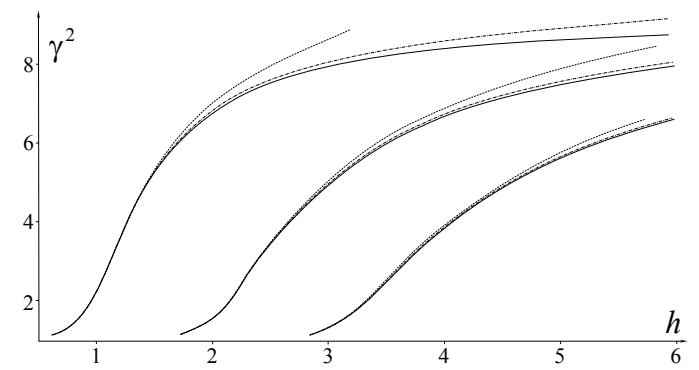

Figure 2. Dispersion curves of the problem.

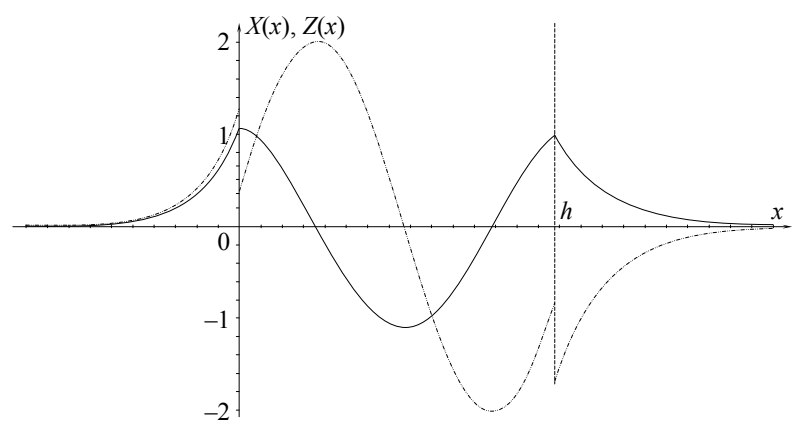

Figure 3. Eigenfunctions for the second eigenvalue of the problem.

$\left(E_{z}^{(h)}\right)^{2}=1$. In the case of nonlinear dispersion equation, the value $a=0.1$ is employed.

For the next case the following initial data are chosen: $\varepsilon_{1}=1, \quad \varepsilon_{2}=4, \quad \varepsilon_{3}=2,\left(E_{z}^{(h)}\right)^{2}=1, \quad a=0.01$ and $h=5.91$. In this case there are three eigenvalues $\gamma_{1}, \gamma_{2}$ and $\gamma_{3}: \gamma_{1}^{2}=3.9, \gamma_{2}^{2}=3.108$ and $\gamma_{3}^{2}=2.1711$.

In Figure 3 eigenfunctions $X(x)$ and $Z(x)$ corresponding to the second eigenvalue $\gamma_{2}$ are depicted. The dash-dotted line corresponds to the eigenfunction $X(x)$ and the solid line corresponds to the eigenfunction $Z(x)$.

It is necessary to note that the component $X(x)$ is not continuous at the points $x=0$ and $x=h$ (at the interfaces) and it has finite jumps at these points. The component $Z(x)$ is continuous but not differentiable at the points $x=0$ and $x=h$.

\section{References}

[1] P. N. Eleonskii, L. G. Oganes'yants and V. P. Silin, "Cylindrical Nonlinear Waveguides," Soviet Physics-Journal of Experimental and Theoretical Physics, Vol. 35, No. 1,
1972, pp. 44-47.

[2] R. I. Joseph and D. N. Christodoulides, "Exact Field Decomposition for TM Waves in Nonlinear Media," Optics Letters, Vol. 12, No. 10, 1987, pp. 826-828.

[3] K. M. Leung, "P-polarized Nonlinear Surface Polaritons in Materials with Intensity-Dependent Dielectric Functions," Physical Review B, Vol. 32, No. 8, 1985, pp. 50935101.

[4] K. M. Leung and R. L. Lin, "Scattering of TransverseMagnetic Waves with a Nonlinear Film: Formal Field Solutions in Quadratures," Physical Review B, Vol. 44, No. 10, 1991, pp. 5007-5012.

[5] H. W. Schürmann, V. S. Serov and Y. V. Shestopalov, "TE-Polarized Waves Guided by a Lossless Nonlinear Three-Layer Structure," Physical Review E, Vol. 58, No. 1, 1998, pp. 1040-1050.

[6] H. W. Schürmann, V. S. Serov and Y. V. Shestopalov, "Solutions to the Helmholtz Equation for TE-Guided Waves in a Three-Layer Structure with Kerr-Type Nonlinearity," Journal of physics A, Mathematical and General, Vol. 35, No. 50, 2002, pp. 10789-10801.

[7] H. W. Schürmann, Y. G. Smirnov and Y. V. Shestopalov, "Propagation of TE-Waves in Cylindrical Nonlinear Dielectric Waveguides," Physical Review E, Vol. 71, No. 1, 2005, pp. 016614(1-10).

[8] Y. G. Smirnov, H. W. Schürmann and Y. V. Schestopalov, "Integral Equation Approach for the Propagation of TE-Waves in a Nonlinear Dielectric Cylinrical Waveguide," Journal of Nonlinear Mathematical Physics, Vol. 11, No. 2, 2004, pp. 256-268.

[9] G. A. Korn and T. M. Korn, "Mathematical Handbook for Scientists and Engineers," McGraw Hill Book Company, 1968.

[10] H. F. Baker, "Abelian Functions. Abel's Theorem and the Allied Theory of Theta Functions," Cambridge University Press, Cambridge, 1897.

[11] A. I. Markushevich, "Introduction to the Classical Theory of Abelian Functions," American Mathematical Society, Providence, 2006.

[12] I. T. Gokhberg and M. G. Krein, "Introduction in the Theory of Linear Nonselfadjoint Operators in Hilbert Space," American Mathematical Society, Providence, 1969.

[13] A. Snyder and J. Love, "Optical Waveguide Theory," Chapmen and Hall, London, 1983.

[14] D. V. Valovik and Y. G. Smirnov, "Calculation of the Propagation Constants and Fields of Polarized Electromagnetic TM Waves in a Nonlinear Anisotropic Layer," Journal of Communications Technology and Electronics, Vol. 54, No. 4, 2009, pp. 391-398.

[15] D. V. Valovik and Y. G. Smirnov, "Calculation of the Propagation Constants of TM Electromagnetic Waves in a Nonlinear Layer," Journal of Communications Technology and Electronics, Vol. 53, No. 8, 2008, pp. 883-889. 